\title{
Non-perturbative quark mass dependence in the heavy-light sector of two-flavour QCD
}

\section{A $L P H A$ \\ Collaboration}

CERN-PH-TH/2008-207

MS-TP-08-25

DESY 08-148

SFB/CPP-08-85

\section{Michele Della Morte}

CERN, Physics Department, TH Unit, CH-1211 Geneva 23, Switzerland

E-mail: dellamor@mail.cern.ch

Patrick Fritzsch* ${ }^{*}$, Jochen Heitger

Westfälische Wilhelms-Universität Münster, Institut für Theoretische Physik,

Wilhelm-Klemm-Str. 9, D-48149 Münster, Germany

E-mail: fritzscheuni-muenster.de, heitgereuni-muenster.de

\section{Rainer Sommer}

Deutsches Elektronen-Synchrotron DESY, Zeuthen,

Platanenallee 6, D-15738 Zeuthen, Germany

E-mail: rainer.sommer@desy.de

\begin{abstract}
We present preliminary results of the non-perturbative heavy quark mass dependence of heavylight meson observables in the continuum limit of finite-volume two-flavour lattice QCD. These observables, which are derived from heavy-light Schrödinger functional correlation functions and computed over a range of renormalization group invariant heavy quark masses from the charm to beyond the bottom region, allow for a quantitative comparison with the predictions of HQET and are of practical relevance for solving renormalization problems in HQET non-perturbatively by a matching to QCD in finite volume.
\end{abstract}

The XXVI International Symposium on Lattice Field Theory

July 14 - 19, 2008

Williamsburg, Virginia, USA

\footnotetext{
* Speaker.

${ }^{\dagger}$ Present address: School of Physics and Astronomy, University of Southampton, Highfield, Southampton, SO17 1BJ, UK
} 


\section{Introduction}

The Heavy Quark Effective Theory (HQET) Lagrangian,

$$
\mathscr{L}_{\mathrm{HQET}}(x)=\bar{\psi}_{\mathrm{h}}(x)\left[D_{0}+m-\omega_{\mathrm{kin}} \mathbf{D}^{2}-\omega_{\mathrm{spin}} \sigma \mathbf{B}\right] \psi_{\mathrm{h}}(x)+\mathrm{O}\left(1 / m^{2}\right),
$$

provides an expansion of QCD amplitudes in the inverse heavy quark mass, $1 / m$, and is renormalizable at any finite order in $1 / m$ by means of power counting. It is a standard phenomenological tool which simplifies the QCD dynamics in the limit of large masses like that of the c- or b-quark. The HQET Lagrangian as written in (1.1) consists of a leading, static term, $D_{0}$, which describes the dynamics in the limit of infinitely heavy quark mass, and of the two subleading kinetic and spin terms whose coefficients are $\omega_{\text {kin }}=\omega_{\text {spin }}=1 /(2 m)$ in the classical theory.

To explicitly make HQET an effective theory of QCD requires matching calculations to express the parameters in the Lagrangian $\left(m, \omega_{\mathrm{kin}}, \cdots\right)$ by those of QCD. In principle one could employ perturbation theory in the matching step, but owing to the difficulty of a reliable error estimation it may be hard to disentangle and quantify deviations coming from higher orders or non-perturbative effects in particular, since there are power-divergent mixings [1]. Despite its phenomenological success - reflecting, e.g., in determinations of $V_{\mathrm{cb}}$ [2, 3] or HQET hadronic matrix elements where perturbative HQET enters - independent and non-perturbative tests of HQET may provide a deeper insight into the feasibility of the effective theory approach. In the quenched approximation, such a test was performed and discussed in [4].

In the course of the non-perturbative matching between QCD and HQET, originally proposed in [5] and currently being applied to QCD with two (massless) dynamical sea quarks [6] (see [7] for the variant of the HMC algorithm we use), we here give a preliminary report on an extension of the quenched study [4] to the two-flavour case. The non-perturbative large-mass behaviour of some meson observables, computed in the continuum limit of finite-volume QCD, is confronted with the static theory in order to investigate the range of validity of the $1 / \mathrm{m}$-expansion and to estimate the size of the $1 / m$-corrections. The use of a finite volume of about $(0.5 \mathrm{fm})^{4}$ is crucial in this context, since clean non-perturbative comparisons of QCD and HQET in the continuum limit require $m \ll 1 / a$ prior to $a \rightarrow 0$.

\section{Observables and their large-mass asymptotics}

Our observables are built from relativistic heavy-light Schrödinger functional (SF) correlation functions. $f_{\mathrm{A}}$ is a correlator between a heavy-light pseudoscalar boundary source and an axial current operator insertion in the bulk, $f_{1}$ a boundary-to-boundary correlation function and $k_{\mathrm{V}}, k_{1}$ are their vector channel analogues. We further use the pseudoscalar, $f_{\mathrm{P}}$, and tensor, $k_{\mathrm{T}}$, correlators to improve the bare currents in the respective channels. More details and any unexplained notation are found in [4]. From now on, these correlators are referred to as their $\mathrm{O}(a)$ improved lattice versions, which amounts to replace the quark bilinears such as the heavy-light axial current according to

$$
A_{\mu} \rightarrow Z_{\mathrm{A}}\left[1+\frac{1}{2} b_{\mathrm{A}}\left(a m_{\mathrm{q}, 1}+a m_{\mathrm{q}, \mathrm{h}}\right)\right] \times A_{\mu} .
$$


We take the non-perturbative values of $Z_{\mathrm{A}}, Z_{\mathrm{V}}$ from [8,9] and the 1-loop perturbative estimates of [10] for $b_{\mathrm{X}}, \mathrm{X} \in\{\mathrm{A}, \mathrm{V}\}$. Our test observables built from these renormalized correlators are

$$
\begin{aligned}
\Gamma_{\mathrm{av}}(L, M) & \equiv \frac{1}{4}\left[\Gamma_{\mathrm{PS}}(L, M)+3 \Gamma_{\mathrm{V}}(L, M)\right], & R_{\mathrm{spin}}(L, M) & \equiv \frac{1}{4} \ln \left(f_{1} / k_{1}\right), \\
Y_{\mathrm{PS}}(L, M) & \equiv+\frac{f_{\mathrm{A}}(T / 2)}{\sqrt{f_{1}}}, & Y_{\mathrm{V}}(L, M) & \equiv-\frac{k_{\mathrm{V}}(T / 2)}{\sqrt{k_{1}}}, \\
R_{\mathrm{PS} / \mathrm{V}}(L, M) & \equiv-\frac{f_{\mathrm{A}}(T / 2)}{k_{\mathrm{V}}(T / 2)}, & R_{\mathrm{PS} / \mathrm{P}}(L, M) & \equiv-\frac{f_{\mathrm{A}}(T / 2)}{f_{\mathrm{P}}(T / 2)},
\end{aligned}
$$

where the following definitions of the pseudoscalar and vector effective energies apply:

$$
\begin{gathered}
\Gamma_{\mathrm{PS}}(L, M) \equiv-\left.\frac{\mathrm{d}}{\mathrm{d} x_{0}} \ln \left[f_{\mathrm{A}}\left(x_{0}\right)\right]\right|_{x_{0}=T / 2}=-\frac{f_{\mathrm{A}}^{\prime}(T / 2)}{f_{\mathrm{A}}(T / 2)} \\
\Gamma_{\mathrm{V}}(L, M) \equiv-\left.\frac{\mathrm{d}}{\mathrm{d} x_{0}} \ln \left[k_{\mathrm{V}}\left(x_{0}\right)\right]\right|_{x_{0}=T / 2}=-\frac{k_{\mathrm{V}}^{\prime}(T / 2)}{k_{\mathrm{V}}(T / 2)}
\end{gathered}
$$

In physically large volume, as $L \rightarrow \infty, \Gamma_{\text {av }}$ becomes proportional to the spin-averaged mass of the heavy-light meson, $R_{\text {spin }}$ to the spin-splitting term and $Y_{\mathrm{PS}}\left(Y_{\mathrm{V}}\right)$ to the pseudoscalar (vector) heavy-light meson decay constant; in this sense we will also use the shorthand decay constant.

These quantities are expected to be described by a power series in $1 / z$ with logarithmic modifications, where

$$
\begin{aligned}
& z=L_{1} M \\
& M=\lim _{\mu \rightarrow \infty}\left\{\left[2 b_{0} \bar{g}^{2}(\mu)\right]^{-d_{0} /\left(2 b_{0}\right)} \bar{m}(\mu)\right\}, \\
& N_{\mathrm{f}}=2 \text {, } \\
& b_{0}=\left(11-\frac{2}{3} N_{\mathrm{f}}\right) /(4 \pi)^{2}, \quad d_{0}=8 /(4 \pi)^{2},
\end{aligned}
$$

and $M$ denotes the renormalization group invariant (RGI) mass of the heavy quark flavour. Our choice of lattices with spatial extent $L=L_{1}, T=L$, and the corresponding simulation parameters are summarized in table 1 . In physical units, $L_{1}$ is about $0.5 \mathrm{fm}$. For a more detailed account on how the $z$-values under investigation, $z \in\{4,6,7,9,11,13,15,18,21\}$, can be kept fixed for given $L_{1} / a$ in dependence of the bare parameters, the reader is referred to [6].

As we are interested in the large $z$-asymptotics of our observables, one also needs their counterparts computed in the effective theory, as long as the latter are non-trivial. We denote the associated quantities by a superscript 'stat', e.g. $f_{\mathrm{A}} \rightarrow f_{\mathrm{A}}^{\text {stat }}$. In case of the decay constant we then construct

$$
X(L) \equiv \frac{f_{\mathrm{A}}^{\text {stat }}(T / 2)}{\sqrt{f_{1}^{\text {stat }}}}
$$

so that

$$
\lim _{z \rightarrow \infty} Y_{\mathrm{PS}}(L, M)=X(L)=\lim _{z \rightarrow \infty} Y_{\mathrm{V}}(L, M)
$$

holds at the classical level. Due to the heavy-quark spin symmetry in the static limit, $Y_{\mathrm{PS}}$ and $Y_{\mathrm{V}}$ converge to the same limit.

In quantum theory, the scale dependent renormalization of the effective theory introduces the mentioned logarithmic modifications. An example is the axial current in the effective theory, where 


\begin{tabular}{cccc}
\hline$L_{1} / a$ & $\beta$ & $\kappa_{1} \approx \kappa_{\mathrm{c}}$ & $L_{1} m_{\mathrm{l}}$ \\
\hline 20 & 6.1906 & 0.135997290 & $+0.00055(13)$ \\
24 & 6.3158 & 0.135772110 & $-0.000145(66)$ \\
32 & 6.5113 & 0.135421494 & $+0.000143(36)$ \\
40 & 6.6380 & 0.135192285 & $+0.000024(24)$ \\
\hline
\end{tabular}

Table 1: Simulation parameters that correspond to a line of constant physics characterized by $\bar{g}^{2}\left(L_{1}\right)=4.484$ and $L_{1} m_{1} \approx 0$ in the light (i.e. sea) quark sector. Suitable choices for the hopping parameter of the heavy quark, $\kappa_{\mathrm{h}}$, allow to fix its dimensionless RGI mass, $z=L_{1} M$, to a set of desired values extending from the charm to the bottom quark region. See [6] for details.

the renormalized $X_{\mathrm{R}}(L, \mu)=Z_{\mathrm{A}}^{\text {stat }}(\mu) X_{\text {bare }}(L)$ depends logarithmically on the chosen renormalization scale $\mu$ as well as on the renormalization scheme. As for the mass, eq. (2.7), this dependence is removed explicitly by passing to the RGI matrix element

$$
X_{\mathrm{RGI}}(L)=\lim _{\mu \rightarrow \infty}\left\{\left[2 b_{0} \bar{g}^{2}(\mu)\right]^{-\gamma_{0} /\left(2 b_{0}\right)} X_{\mathrm{R}}(L, \mu)\right\}=Z_{\mathrm{RGI}}^{\text {stat }} X_{\text {bare }}(L), \quad \gamma_{0}=-1 /\left(4 \pi^{2}\right) .
$$

For $N_{\mathrm{f}}=2$, the renormalization factor $Z_{\mathrm{RGI}}^{\text {stat }}$ is known non-perturbatively from [11]. After expressing our QCD test observables through the corresponding RGIs, their large- $z$ behaviour is driven by the RGIs of the effective theory together with so-called conversion functions, $C$, which contain the full logarithmic mass dependence of the associated operators. As arguments of the latter we choose the ratio of RGIs $M / \Lambda$, since it can be fixed on the lattice without perturbative uncertainties [12].

The $1 / z$-expansions of our test observables now read as follows:

$$
\begin{array}{rll}
Y_{\mathrm{X}}(L, M) & \stackrel{M \rightarrow \infty}{\sim} C_{\mathrm{X}}\left(M / \Lambda_{\overline{\mathrm{MS}}}\right)\left[X_{\mathrm{RGI}}(L)\right](1+\mathrm{O}(1 / z)), & \mathrm{X}=\mathrm{PS}, \mathrm{V}, \\
R_{\mathrm{X}}(L, M) & \stackrel{M \rightarrow \infty}{\sim} C_{\mathrm{X}}\left(M / \Lambda_{\overline{\mathrm{MS}}}\right)[1](1+\mathrm{O}(1 / z)), & \mathrm{X}=\mathrm{PS} / \mathrm{V}, \mathrm{PS} / \mathrm{P}, \\
R_{\mathrm{spin}}(L, M) & \stackrel{M \rightarrow \infty}{\sim} C_{\text {spin }}\left(M / \Lambda_{\overline{\mathrm{MS}}}\right)\left[X_{\mathrm{RGI}}^{\text {spin }}(L) / z\right](1+\mathrm{O}(1 / z)), & \\
L \Gamma_{\mathrm{av}}(L, M) & \stackrel{M \rightarrow \infty}{\sim} C_{\text {mass }}\left(M / \Lambda_{\overline{\mathrm{MS}}}\right)[z](1+\mathrm{O}(1 / z)) . &
\end{array}
$$

Beside the overall logarithmic mass dependence governed by the conversion functions we enclose the leading matrix elements in the effective theory in square brackets. The numerical evaluation of the $C_{\mathrm{X}}$ proceeds as explained in $[4,11]$, where always the highest available perturbative approximation of the involved RG functions is employed, in particular the 3-loop anomalous dimensions $\gamma$ of the axial current and the chromomagnetic operator in HQET, respectively [13, 14]. By comparing different loop orders for their evaluation, we conclude that the remaining perturbative uncertainty of the conversion functions originating from unknown higher orders is much smaller than the precision of our lattice data so that a study of the $1 / z^{n}$-corrections becomes feasible.

\section{Results}

As examples for the observables under study, continuum extrapolations linear in $(a / L)^{2}$ of $L \Gamma_{\mathrm{av}}$ and $R_{\text {spin }}$ for the phase parameter ${ }^{1} \theta=0.5$ are presented in fig. 1 . In our volume of extent

\footnotetext{
${ }^{1}$ The SF boundary conditions in space on the fermion fields are taken to be periodic up to a global phase $\theta$.
} 

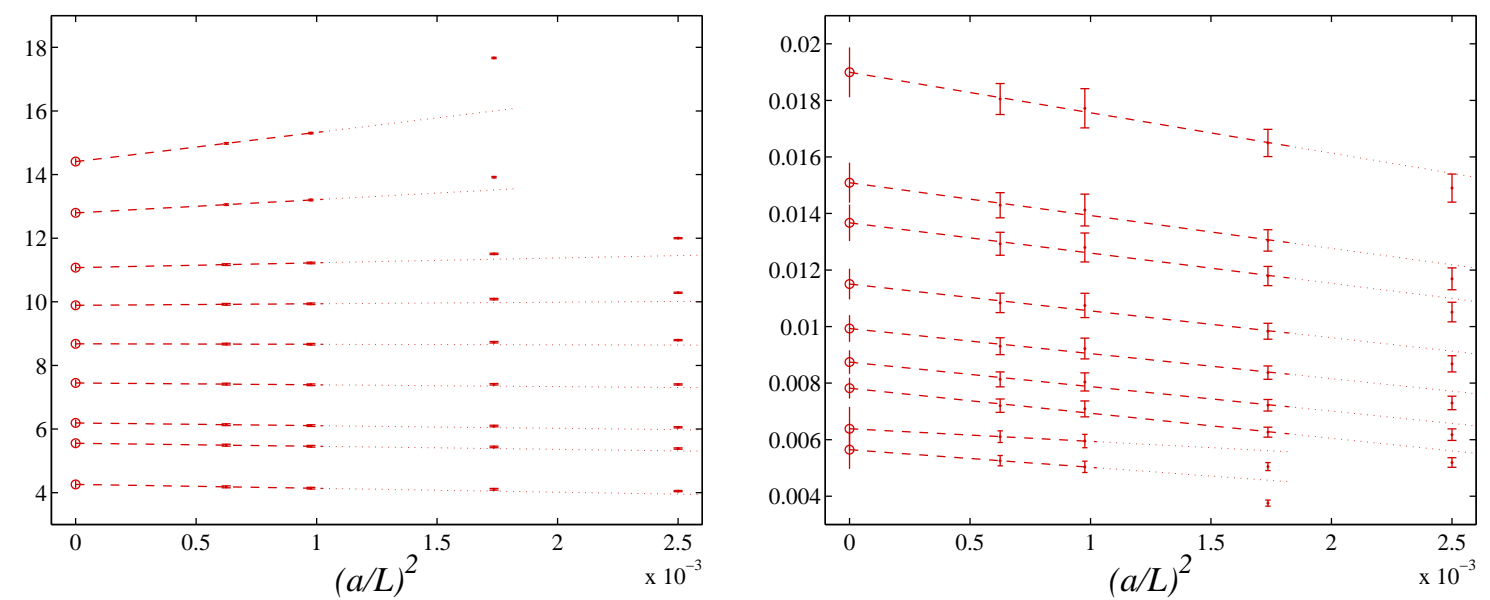

Figure 1: Left: Continuum extrapolations linear in $(a / L)^{2}$ of the spin-averaged mass $L \Gamma_{\mathrm{av}}(z, \theta=0.5)$ from $z=4$ (bottom) to $z=21$ (top). The error of the continuum limits is smaller than the symbol size. Right: Continuum extrapolations linear in $(a / L)^{2}$ of the spin-splitting $R_{\text {spin }}(z, \theta=0.5)$ from $z=4$ (top) to $z=21$ (bottom). - Depending on the value of $z$, the coarsest or the coarsest two lattices are omitted from the fits.

$L=L_{1}=T \approx 0.5 \mathrm{fm}$, which admits to reach heavy quark masses up to $M \approx 1.3 M_{\mathrm{b}}$, the $a \rightarrow 0$ extrapolations appear to be well controllable provided that one accounts for the growing (heavy) quark mass in lattice units at given $a / L$ as $z$ is increased. Similar to the quenched work [4], this is realized by imposing a cut on $a M(a M \lesssim 0.7)$ that translates, for given $z$, into the coarsest resolutions which may still be included in the continuum extrapolations. For all observables considered, the growing deviation, as $z$ is increased, of the extrapolating fit function from the result at the respective coarsest lattice resolution available suggests that between $z=18$ and $z=21$ the $\mathrm{O}(a)$ improvement and thus the $a$-expansion has broken down for our lattices.

Polynomial fits in $1 / z$ of the continuum $L \Gamma_{\mathrm{av}} /\left(z C_{\text {mass }}\right),\left(z R_{\text {spin }}\right) / C_{\text {spin }}$ as well as of the finitevolume pseudoscalar and vector decay constants and their ratio $R_{\mathrm{PS} / \mathrm{V}}$ (divided by the corresponding conversion functions) are shown in figs. 2 and 3. If the conversion functions $C_{\mathrm{X}}$ are evaluated including the highest available perturbative accuracy, the $z$-dependence of $\Gamma_{\mathrm{av}}$ and $R_{\mathrm{PS} / \mathrm{v}}$ is consistent with the leading term in the $1 / z$-expansion, which in case of $R_{\mathrm{PS} / \mathrm{V}}$ is fixed by the heavy-quark spin symmetry of the static theory. Note in addition that the $1 / z \rightarrow 0$ limit of these observables is independent of the choice for the periodicity angle $\theta(=0,0.5,1$ here $)$, as it should be.

In general, the $1 / z$-corrections of our observables are reasonably small. As can be inferred from the 1.h.s. of fig. 3, $Y_{\mathrm{PS}} / C_{\mathrm{PS}}$ and $Y_{\mathrm{V}} / C_{\mathrm{V}}$ converge to the same limit (see (2.9)) as expected. In principle, this common limit of their $z$-dependence can be constrained by the static theory as well, because the renormalization factor of the associated static axial current matrix element, (2.10), is already known non-perturbatively [11]. The computation of the bare matrix element to obtain the static result for $X_{\mathrm{RGI}}$ is in progress.

\section{Conclusions}

We have presented a status report of our ongoing study of the large-mass asymptotics of nonperturbatively renormalized heavy-light meson observables in finite-volume two-flavour QCD. 

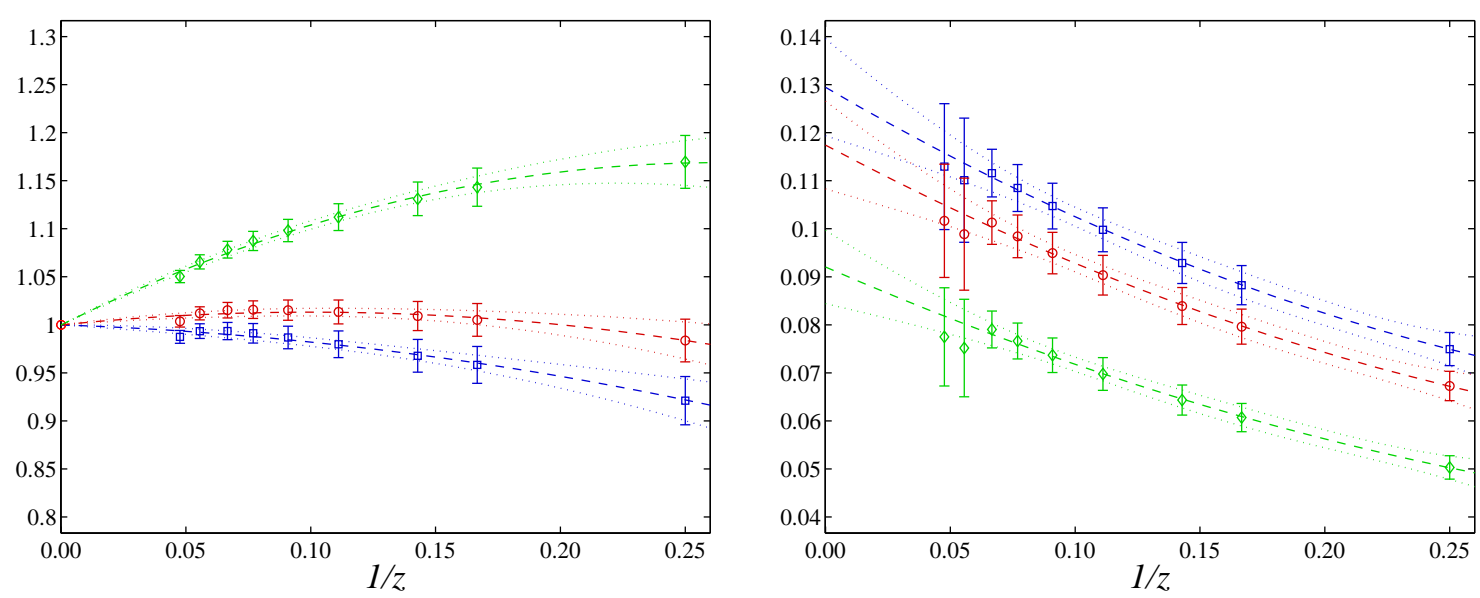

Figure 2: Left: Asymptotics of $L \Gamma_{\mathrm{av}} /\left(z C_{\text {mass }}\right)$ versus $1 / z$. All data sets are extrapolated to the (known) static limit (=1, cf. (2.14)) with a constrained quadratic fit. Right: $1 / z$-dependence of $\left(z R_{\text {spin }}\right) / C_{\text {spin }}$ with an unconstrained quadratic fit to all data points. - Blue, red and green symbols refer to $\theta \in\{0,0.5,1\}$.
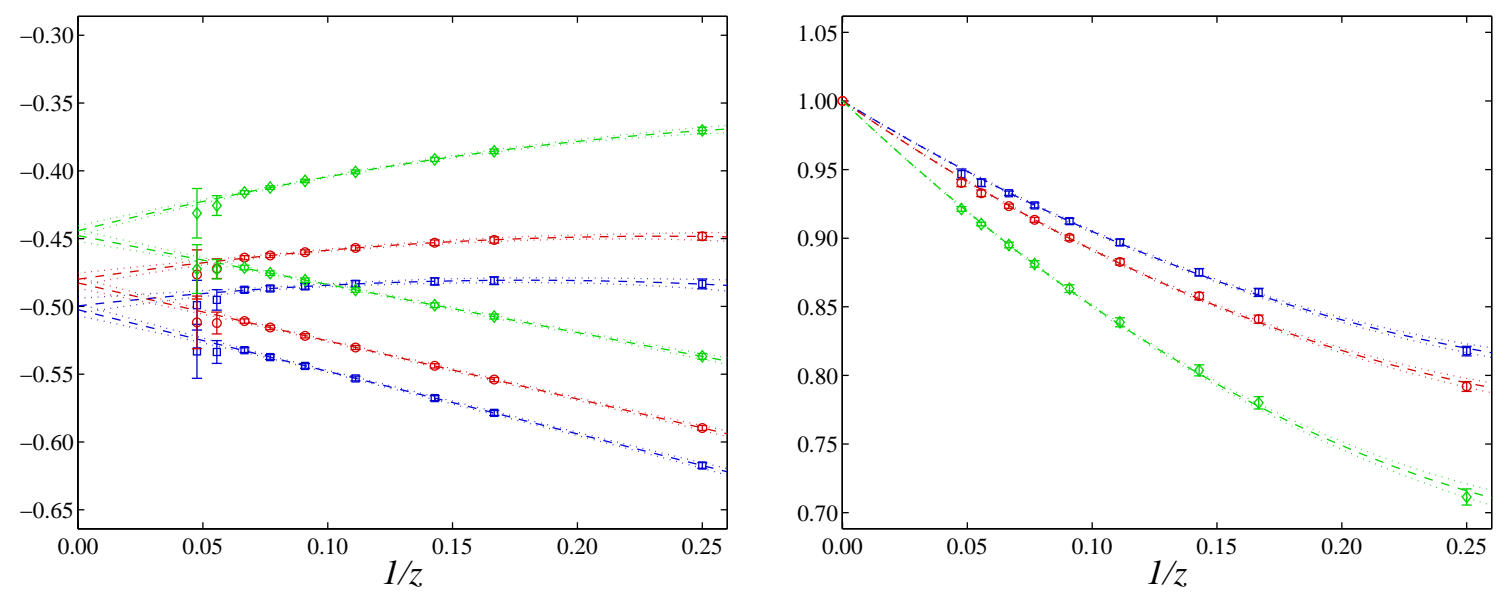

Figure 3: Left: Asymptotics of $Y_{\mathrm{X}} / C_{\mathrm{X}}$ versus $1 / z$ and its unconstrained quadratic fits to the static limit. Data sets approaching $1 / z=0$ from above (below) corresponds to $\mathrm{X}=\mathrm{PS}(\mathrm{X}=\mathrm{V})$. Right: $R_{\mathrm{PS} / \mathrm{V}} / C_{\mathrm{PS}} / \mathrm{v}$ versus $1 / z$ and constrained quadratic fits to the static limit (=1, cf. (2.12)).

Their behaviour in dependence of the inverse RGI heavy quark mass is well compatible with the predictions of HQET confirming the feasibility of a precise non-perturbative matching of QCD and HQET. The only perturbative uncertainties owing to the leading logarithmic mass dependence, which is induced by the conversion functions $C$ relating our observables to the RGIs of the effective theory, are under reasonable control. It appears that for the studied observables the power corrections dominate over the perturbative ones in the considered range of $z$.

Our continuum extrapolations may be further improved by a removal of perturbative cutoff effects prior to the extrapolations.

\section{Acknowledgments}

This work is part of our effort for precision B-physics with $N_{\mathrm{f}}=2$. We would like to thank our colleagues B. Blossier, G. de Divitiis, N. Garron, G. von Hippel, R. Petronzio, H. Simma and 
N. Tantalo for discussions and collaboration in this effort. We thank NIC for allocating computer time on the APE computers to this project and the APE group at Zeuthen for support. We further acknowledge partial support by the Deutsche Forschungsgemeinschaft under grant HE 4517/2-1 and in the SFB/TR 09-03, "Computational Particle Physics", as well as by the European Community through EU Contract No. MRTN-CT-2006-035482, "FLAVIAnet". P.F. would like to thank the Lattice 2008 organizing committee for financial support of his participation in the conference.

\section{References}

[1] L. Maiani, G. Martinelli and C. T. Sachrajda, Nonperturbative subtractions in the heavy quark effective field theory, Nucl. Phys. B368 (1992) 281.

[2] Particle Data Group Collaboration, C. Amsler et. al., Review of particle physics, Phys. Lett. B667 (2008) 1 [http://pdg.1bl.gov].

[3] G. M. de Divitiis, R. Petronzio and N. Tantalo, Quenched lattice calculation of the vector channel $B \rightarrow D^{*} \ell v$ decay rate, arXiv:0807.2944 [hep-lat ].

[4] ALPHA Collaboration, J. Heitger, A. Jüttner, R. Sommer and J. Wennekers, Non-perturbative tests of heavy quark effective theory, J. High Energy Phys. 11 (2004) 048 [hep-ph/ 0407227 ].

[5] ALPHA Collaboration, J. Heitger and R. Sommer, Non-perturbative heavy quark effective theory, J. High Energy Phys. 02 (2004) 022 [hep-lat/ 0310035$].$

[6] ALPHA Collaboration, M. Della Morte, P. Fritzsch, J. Heitger, H. B. Meyer, H. Simma and R. Sommer, Towards a non-perturbative matching of HQET and QCD with dynamical light quarks, PoS LAT2007 (2007) 246 [arXiv: 0710.1188 [hep-lat] ].

[7] ALPHA Collaboration, H. B. Meyer, H. Simma, R. Sommer, M. Della Morte, O. Witzel and $\mathrm{U}$. Wolff, Exploring the HMC trajectory-length dependence of autocorrelation times in lattice QCD, Comput. Phys. Commun. 176 (2007) 91 [hep-lat/ 0606004 ].

[8] ALPHA Collaboration, M. Della Morte, R. Sommer and S. Takeda, On cutoff effects in lattice QCD from short to long distances, arXiv:0807.1120 [hep-lat] .

[9] ALPHA Collaboration, M. Della Morte, R. Hoffmann, F. Knechtli, R. Sommer and U. Wolff, Non-perturbative renormalization of the axial current with dynamical Wilson fermions, J. High Energy Phys. 07 (2005) 007 [hep-lat/ 0505026 ].

[10] ALPHA Collaboration, S. Sint and P. Weisz, Further results on $O(a)$ improved lattice QCD to one-loop order of perturbation theory, Nucl. Phys. B502 (1997) 251 [hep-lat/9704001].

[11] ALPHA Collaboration, M. Della Morte, P. Fritzsch and J. Heitger, Non-perturbative renormalization of the static axial current in two-flavour QCD, J. High Energy Phys. 02 (2007) 079 [hep-lat/0611036].

[12] ALPHA Collaboration, M. Della Morte, R. Hoffmann, F. Knechtli, J. Rolf, R. Sommer, I. Wetzorke and U. Wolff, Non-perturbative quark mass renormalization in two-flavor QCD, Nucl. Phys. B729 (2005) 117 [hep-lat/0507035].

[13] K. G. Chetyrkin and A. G. Grozin, Three-loop anomalous dimension of the heavy-light quark current in HQET, Nucl. Phys. B666 (2003) 289 [hep-ph/ 0303113$].$

[14] A. G. Grozin, P. Marquard, J. H. Piclum and M. Steinhauser, Three-Loop Chromomagnetic Interaction in HQET, Nucl. Phys. B789 (2008) 277-293 [arXiv:0707.1388 [hep-ph]]. 